\title{
Análise temporal da relação entre leptospirose e ocorrência de inundações por chuvas no município do Rio de Janeiro, Brasil, 2007-2012
}

\author{
Temporal analysis of the relationship between leptospirosis \\ and the occurrence of flooding due to rainfall in the city \\ of Rio de Janeiro, Brazil, 2007-2012
}

\author{
Raphael Mendonça Guimarães ${ }^{1}$ \\ Oswaldo Gonçalves Cruz ${ }^{2}$ \\ Viviane Gomes Parreira ${ }^{3}$ \\ Maíra Lopes Mazoto ${ }^{1}$ \\ Juliana Dias Vieira ${ }^{3}$ \\ Carmen Ildes Rodrigues Fróes Asmus ${ }^{1}$
}

${ }^{1}$ Instituto de Estudos em Saúde Coletiva, Universidade Federal do Rio de Janeiro. Av. Horácio Macedo s/n, Fundão. 21.941-598 Rio de Janeiro RJ Brasil. raphael@iesc.ufrj.br ${ }^{2}$ Programa de Computação Científica, Fundação Oswaldo Cruz.

${ }^{3}$ Secretaria Municipal de Saúde e Defesa Civil do Rio de Janeiro.

\begin{abstract}
The scope of this study is to examine the effect of the average monthly rainfall on the risk of contracting leptospirosis in the city of Rio de Janeiro between 2007 and 2012. It involves an analytical ecological study conducted in the city of Rio de Janeiro in the 2007-2012 period. The variable for the outcome was the number of leptospirosis cases per month, and the independent variable was the average monthly rainfall recorded by the 32 monitoring stations per year. It was decided to model the relationship between cases of leptospirosis and the effects of rain by building a generalized linear model using negative binomial distribution. The rainfall-lag per month was found to be a strong explanatory factor for the number of cases of leptospirosis. This study indicates that the average monthly rainfall may constitute an indicator that enables the execution of actions in order to prepare the health sector for the probable increase in cases of this disease. Therefore, it is necessary to implement the interaction between the work of epidemiological surveillance, especially by situation rooms in periods of crisis, and the risk management teams of environmental surveillance, to increase the response capacity to natural disasters in the municipality of Rio de Janeiro Key words Leptospirosis, Climate, Floods, Temporal distribution
\end{abstract}

Resumo O objetivo do presente estudo é examinar o efeito da média mensal de precipitações sobre o risco de leptospirose na cidade do Rio de Janeiro entre 2007 e 2012. Trata-se de um estudo ecológico analítico cuja variável de desfecho foi o número de casos de leptospirose por mês, e a variável independente foi a precipitação média mensal para as 32 estações de monitoramento por ano. Optou-se por modelar a relação entre os casos de leptospirose e o efeito da chuva através da construção de um modelo linear generalizado, utilizando a distribuição binomial negativa. A precipitação-lag mês mostrou ser um fator explicativo forte para o número de casos de leptospirose. Este estudo indica que a média mensal de precipitações pode se constituir em um indicador que permita a realização de ações visando a preparação do setor saúde para o provável aumento de casos desta doença. Cabe, portanto, a articulação entre o trabalho da vigilância epidemiológica, especialmente aquele feito pelas salas de situação em períodos de crise, e as instâncias de gestão de riscos da vigilância ambiental, para aumentar a capacidade de resposta a desastres naturais no município do Rio de Janeiro.

Palavras-chave Leptospirose, Clima, Inundações, Distribuição Temporal 


\section{Introdução}

A leptospirose é uma doença bacteriana aguda, de distribuição global, que pode manifestar-se de maneira assintomática, por quadros leves ou casos graves que podem levar a morte. A bactéria é eliminada para o meio ambiente principalmente através da urina de animais infectados. Sua ocorrência está classicamente relacionada à exposição aos fatores de risco como situações ocupacionais específicas, precárias condições de infraestrutura sanitária, alta infestação de roedores infectados e, de forma mais ampla, na ocorrência de enchentes $^{1}$. A infecção humana resulta da exposição direta ou indireta à urina de animais infectados. A penetração do microrganismo pode ocorrer através da pele com presença de lesões, pele íntegra imersa por longos períodos em água contaminada ou através de mucosas. O contato com água e lama contaminadas demonstra a importância do elo hídrico na transmissão da doença ao homem² .

No início de sua descoberta, a leptospirose era considerada uma patologia de ocorrência esporádica e predominantemente rural. Atualmente, pode ser encontrada em áreas urbanas, associada principalmente ao crescimento desordenado e áreas de segregação socioeconômica, falha no recolhimento do lixo, existência de terrenos baldios, que propiciam um ambiente favorável à transmissão da doença ${ }^{3}$. Por tudo isso, considerase que a leptospirose é uma zoonose de grande importância social e econômica. Apresenta elevada incidência em determinadas áreas, alto custo hospitalar e perdas de dias de trabalho, bem como por sua letalidade, que pode chegar a até $40 \%$ dos casos mais graves ${ }^{4}$.

Segundo Genovez ${ }^{5}$ a ocorrência de leptospirose está intimamente relacionada a fatores ambientais. As formas mais comuns de se adquirir a doença são em situações de inundações e enchentes, quando a urina dos ratos, presentes em esgotos e bueiros, mistura-se à enxurrada e à lama das enchentes. Assim, na área urbana, especialmente no período chuvoso, as inundaçoes são o principal fator de risco para a ocorrência de surtos da doença.

As inundações são fenômenos históricos no nosso planeta com ocorrências mais antigas do que a existência do próprio homem. No entanto, a frequência e a magnitude de suas ocorrências têm se tornado cada vez maiores em praticamente todo o mundo, especialmente no Brasil, tendo como consequências volumosos prejuízos econômicos e perdas de vidas humanas ${ }^{6}$.

Cerca de 102 milhões de pessoas por ano são atingidas por inundações no mundo. No Brasil, as inundações são o segundo maior desastre natural no Brasil em número de ocorrências, responsáveis por 6.771 ocorrências no período de 1991 a 2010 e 28.600 pessoas afetadas 7 .

Áreas densamente ocupadas têm maior propensão à ocorrência de alagamentos e inundações, principalmente quando a ocupação não foi precedida de planejamento dos sistemas de drenagem das águas pluviais originando uma rede insuficiente para a necessidade de escoamento, em especial nos períodos de grandes precipitações ${ }^{8,9}$. No Município do Rio de Janeiro pode ser observado rotineiramente nas mídias impressas e televisivas que diversas áreas tiveram uma expansão desordenada estando propensas a cheias cíclicas, que propicia a disseminação e a persistência do agente causal no ambiente, facilitando a ocorrência de casos da doença.

Estudos no Brasil ${ }^{1,3,5,6}$ analisaram a leptospirose sob a ótica da distribuição espacial e evidenciaram a relação entre a doença e fatores socioeconômicos através da observação da maior incidência da doença nos estratos de menor infraestrutura sanitária, urbanização e coleta de lixo. Outro fator relacionado com o aumento do número de casos foi a elevação dos índices de precipitação pluviométrica em áreas sujeitas a alagamentos $^{10}$.

As evidências apontam, portanto, para uma relação entre o aumento da precipitação e o aumento das taxas de incidência de leptospirose. Diante disso, o objetivo do presente estudo é examinar o efeito da média mensal de precipitações sobre o risco de leptospirose na cidade do Rio de Janeiro entre 2007 e 2012.

\section{Método}

Trata-se de um estudo ecológico analítico realizado no município do Rio de Janeiro, no período de 2007 a 2012. Optou-se por este desenho de estudo devido ao fato de a variável avaliada (precipitação) ser ecológica, não possuindo uma equivalência mensurável em nível individual.

O município do Rio de Janeiro está localizado na Região Sudeste do Brasil, e possui atualmente uma população estimada de 6.320 .446 habitantes e uma área total de 1.200 quilômetros quadra$\operatorname{dos}^{11}$. A Secretaria Municipal de Saúde do Rio de Janeiro, para fins de planejamento, subdivide o município em 10 Áreas de Planejamento.

Possui grande diversidade geográfica, ambiental e características socioeconômicas, compreendendo áreas densamente ocupadas e flo- 
restas desabitadas, assim como favelas e comunidades em estreita proximidade com bairros de classe média e alta. $\mathrm{O}$ volume pluviométrico acumulado anual fica em torno de $1000 \mathrm{~mm}$, e as chuvas são concentradas nos meses de dezembro a março e afetam regiões com inadequada drenagem de água ${ }^{1}$.

Os casos de leptospirose confirmados em residentes do município do Rio de Janeiro no SINAN (Sistema Nacional de Agravos de Notificação) no período de 2007 a 2012 foram coletados do site da Secretaria Municipal de Saúde do Rio de Janeiro $(\mathrm{SMS} / \mathrm{RJ})^{12}$. Os dados referentes à população por bairro estimados pelo Instituto Brasileiro de Geografia e Estatística (IBGE) foram consultados na página do Departamento de Informática do SUS ${ }^{13}$. Foram obtidos os dados de precipitação acumulada mensal em milímetros de cada uma das 32 estações de monitoramento, no período de dezembro de 2006 a dezembro de 2012, disponíveis no site da Secretaria Municipal de Obras Públicas ${ }^{14}$. Foi escolhido como unidade geográfica de estudo o bairro de residência dos casos, sendo posteriormente agregados em Áreas de Planejamento, e em dois triênios: 2007-2009 e 2010-2012.

Para a elaboração dos mapas foram utilizadas as bases cartográficas digitais do município do Rio de Janeiro, obtidas através do site do Instituto Pereira Passos (IPP Rio) ${ }^{15}$.

A análise exploratória dos dados foi realizada por meio de boxplots, histogramas série tempo de decomposição, e autocorrelação gráfica. A variável de desfecho foi o número de casos de leptospirose por mês, e a variável independente foi a precipitação média mensal para as 32 estações de monitoramento por ano. Optou-se por utilizar a precipitação média ocorrida no mês anterior ao mês de início de sintomas. A associação entre a precipitação e a taxa de incidência de leptospirose foi estimada através do risco relativo para cada mês de observação.

Devido a não existência de autocorrelação temporal nos números de casos, optou-se por modelar a relação entre casos de leptospirose e o efeito da chuva através da construção de um modelo linear generalizado ${ }^{16}$, utilizando a distribuição binomial negativa. Para tratar a sazonalidade, os meses de início de sintomas dos casos foram incorporados ao modelo. Este recurso teve como objetivo considerar para o estudo o tempo de latência da doença, de forma que a variável "caso de leptospirose" foi estimada pelo início dos sintomas, e não pela data de notificação do caso. A Adequação do modelo foi avaliada pelo teste de autocorrelação e normalidade dos resíduos.
As análises foram feitas através do software R, versão 3.02 (R Core Team, 2013), utilizando o pacote $M A S S^{17}$ para a modelagem binomial negativa e os pacotes $s p$ e Maptools ${ }^{18}$ para a criação dos mapas.

\section{Resultados}

Ao analisarmos a distribuição de casos mês a mês observa-se um padrão de sazonalidade da leptospirose, com concentração maior no verão, período em que há também um aumento na precipitação de chuvas. Analisando por intervalos anuais, observa-se na tendência um aumento do número de casos no período 2008-2010, que igualmente é acompanhado pelo aumento da precipitação no mesmo período (Figura 1).

Com base na análise inicial, foi avaliado um modelo cuja variável dependente foi a taxa de incidência de leptospirose, e a variável independente a precipitação de chuvas no Município do Rio de Janeiro. A análise de resíduos mostrou que a correlação máxima se deu no ponto - 1,0 ( $\mathrm{r}=$ $0,57)$, sugerindo que existe de fato um intervalo de tempo entre o pico de precipitação e o surgimento dos sintomas. Desta forma, utilizamos, para análise, o mês anterior da chuva para correlacionar com a ocorrência de casos. Esta escolha é coerente, ainda, com o tempo de latência da doença e, por conseguinte, a inclusão dos casos na semana epidemiológica da vigilância.

Tomando por base o excesso de dispersão dos dados, considerou-se que a distribuição binomial negativa foi uma escolha melhor que a distribuição de Poisson. Visto isso, procedeu-se à análise mês a mês, estimando o risco relativo para a ocorrência da leptospirose e a precipitação de chuvas no mês anterior aos casos. A referência usada foi o mês de agosto, que possui a menor média de chuvas do ano (em milímetros). Observou-se um risco relativo estatisticamente significativo, em ordem decrescente, para os meses de $\operatorname{março}(R R=2,77 ; p=0,005)$, janeiro $(R R=2,71$; $\mathrm{p}=0,007)$, abril $(\mathrm{RR}=2,41 ; \mathrm{p}=0,015)$, dezembro $(R R=2,40 ; p=0,016)$, fevereiro $(R R=2,26$; $\mathrm{p}=0,023)$ e novembro $(\mathrm{RR}=2,14 ; \mathrm{p}=0,032)$ (Figura 2). Ressalta-se que este período corresponde ao período identificado na sazonalidade da Figura 1.

No período analisado, o maior número de casos ocorreu em 2010. Neste período, o município do Rio de Janeiro sofreu o maior episódio de inundação dos últimos 80 anos, o que pode ser atestado pelos picos dos índices de precipita- 

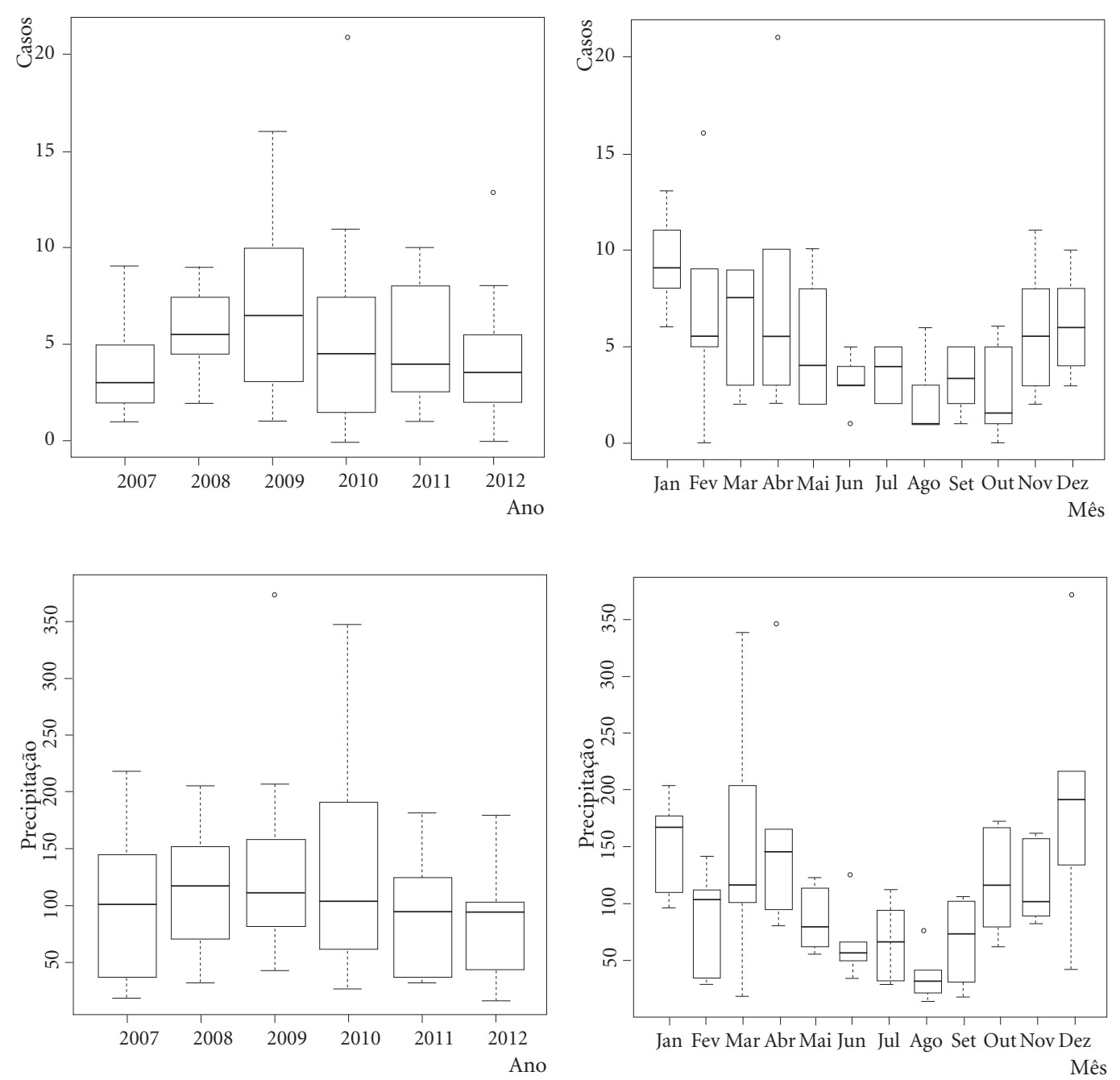

Figura 1. Tendência e sazonalidade das chuvas e casos de leptospirose no Município do Rio de Janeiro, 2007-2012.

ção apresentados. A Figura 3 apresenta as séries temporais de casos e índices de precipitação com um intervalo de 1 mês para os dois eventos, conforme descritos anteriormente. Foi observado, conforme o esperado, que a curva do número de casos, com o lag de um mês, acompanhou a série de precipitação.

Finalmente, ao observar a ocorrência de leptospirose no período, observou-se a densidade de incidência dividida em dois períodos: 2007 a 2009 e 2010 a 2012. A escolha desta divisão se deu usando como critério o pico da incidência da doença e do índice de precipitação de chuvas em 2010 (Figura 4, Tabela 1). Para ambos os triênios, observa-se que a área correspondente à área de planejamento 5.2 apresenta sistematicamente as maiores taxas. Cabe destacar, ainda, para o primeiro triênio, a área 1.0, demonstrando que a dispersão de casos não é uniforme no espaço, demandando, portanto, a necessidade de respostas da vigilância epidemiológica e de desastres de forma diferenciada.

\section{Discussão}

A ocorrência de Leptospirose está intimamente relacionada com o período chuvoso do verão, pois, com o aumento do volume de água, a Leptospira é transportada mais rapidamente pelo 
contato com as águas de inundações urbanas. Além disso, é importante ressaltar o fato de que, neste período de clima quente e úmido, as pessoas têm um maior contato com a água de rios, córregos e lagos em atividades de lazer ${ }^{3}$.

Segundo Aleixo e $\mathrm{Neto}^{3}$ e Magalhães et al. ${ }^{19}$, os casos confirmados de leptospirose podem ser diretamente vinculados às situações de vulnerabilidade socioambiental, sendo portanto um indicador de efeito desta vulnerabilidade, conforme observado no presente estudo. Nos grandes centros urbanos, o intenso e desordenado pro- cesso de urbanização causado pelo rápido crescimento, a falta de saneamento básico e a produção e coleta inadequada de lixo propiciam condições ambientais favoráveis para a reprodução da população de roedores, principais reservatórios da doença ${ }^{8,20}$. Como fator potencializador, fortes chuvas sazonais em regiões tropicais, ao provocarem enchentes e inundações, constituem-se em condição favorável para a exposição ao agente da doença por meio da transmissão hídrica podendo ocasionar epidemias de leptospirose em áreas urbanas ${ }^{8,21-23}$.

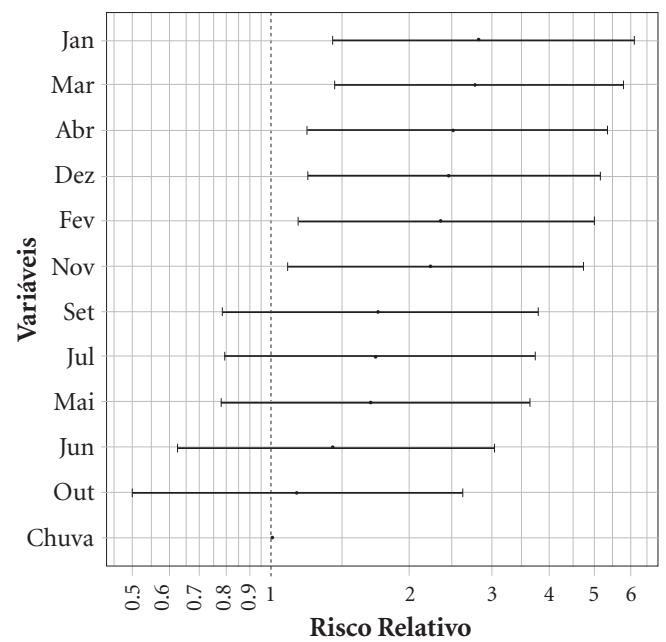

Figura 2. Análise de Risco para leptospirose segundo precipitação mensal no Município do Rio de Janeiro, 2007-2012.

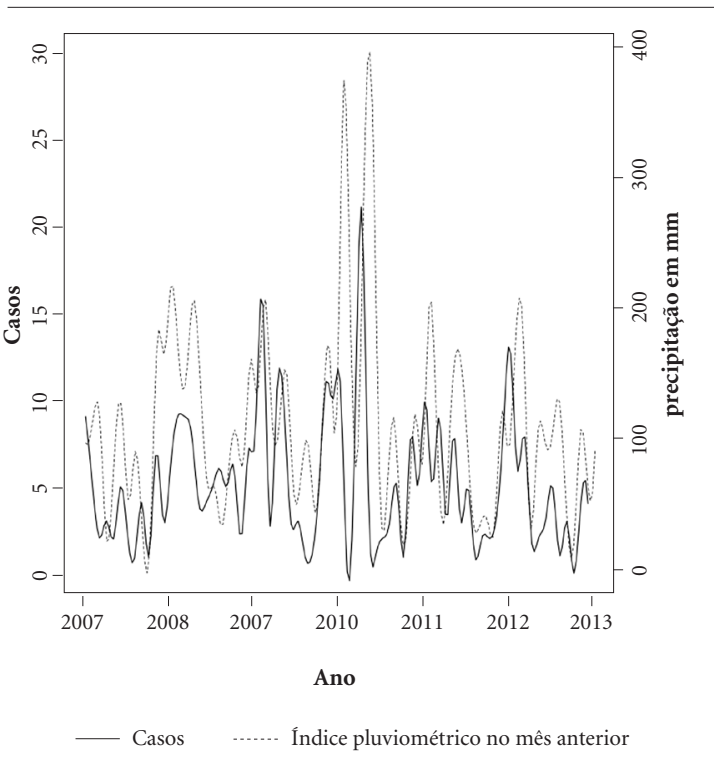

Figura 3. Tendência temporal da precipitação por chuvas e de casos de leptospirose no Município do Rio de Janeiro, 2007-2012.

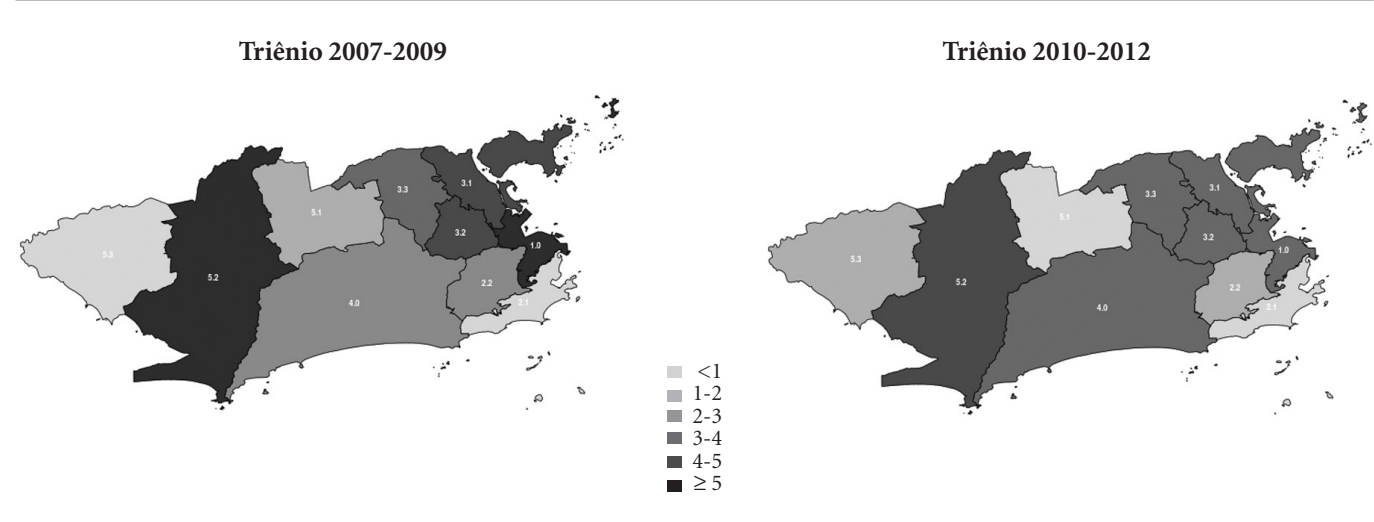

Figura 4. Distribuição espacial das taxas de incidência dos casos de leptospirose no Município do Rio de Janeiro segundo Área de Planejamento, 2007-2012. 


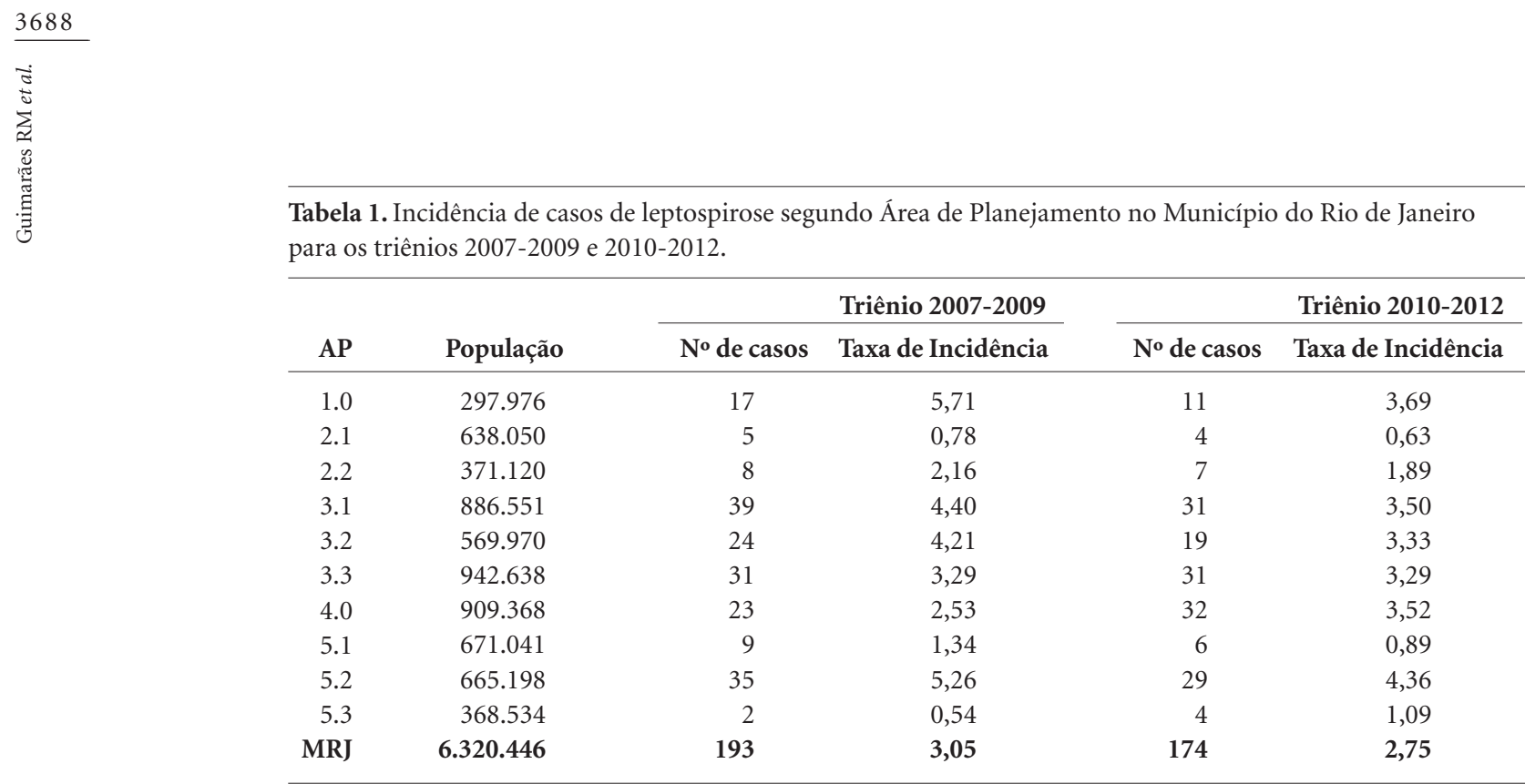

Fonte: SMS/RJ, 2013.

Além disso, é importante considerar que, as alterações ambientais resultantes da destruição das áreas ribeirinhas modificam o curso natural e o fluxo dos rios, facilitando maior extensão das inundações, o que expõe um número maior de pessoas à doença. Outro fator importante é a altitude, pois áreas mais baixas podem concentrar coleções de água ou interferir no curso das águas de enchentes e de desastres naturais ${ }^{21,24}$.

Os estudos de Barcellos e Sabrozza ${ }^{21}$, Pellegri$\mathrm{ni}^{23}$ e Paula ${ }^{25}$ reforçam a estreita associação entre altas precipitações pluviométricas, a ocorrência de inundações e o aumento na incidência de casos de leptospirose no espaço urbano, corroborando os achados do presente estudo, especialmente nos períodos de sazonalidade de chuvas.

Souza et al. ${ }^{26}$ realizaram um estudo cujo objetivo era relacionar o número de ocorrências de leptospirose e sua relação com o regime pluviométrico no município de Belém do Pará. Assim como o presente estudo, eles encontraram uma associação direta entre a incidência da doença e a variação pluviométrica, mostrando que, nos anos com os maiores índices pluviométricos (1998, $1999,2000,2004,2007)$ ocorreu um aumento do número de casos confirmados de leptospirose. A informação que este estudo traz é que, além dos anos de maior pluviosidade, há que se considerar os meses do ano em que a pluviosidade aumenta pelo efeito sazonal das chuvas. Vale ressaltar também que a pluviosidade e o número de casos de leptospirose têm relação direta com a saturação do solo e a urbanização.

Barcellos e Sabroza ${ }^{21}$, por sua vez, realizaram um estudo para investigar o contexto ambiental do surto de leptospirose ocorrido na Zona Oeste do Rio de Janeiro no verão do ano de 1996. Os resultados obtidos pelos autores evidenciaram que as maiores taxas de incidência de leptospirose ocorreram nas regiões sujeitas à inundação $\mathrm{e}$ nas regiões em torno das zonas de acumulação de lixo, reforçando o papel de fatores de risco ambientais na determinação da doença. A mesma relação foi encontrada para o presente estudo, para anos diferentes do estudo de Barcellos e Sabroza $^{21}$, e isto se evidencia na Figura 4 apresentada. A mesma correlação foi observada por Socolovschi et al..$^{27} \mathrm{em}$ estudo realizado na cidade de Marselha, França. Percebeu-se que o aumento de casos da doença na cidade se tornou um problema de saúde pública e estava associado à combinação da ocorrência de forte chuvas e greves das empresas que coletavam lixo, o que contribuía para o aumento do número de ratos na cidade.

Zhang et al. ${ }^{28}$ observaram a prevalência da leptospirose na população chinesa no período de 1955 a 2010. Eles observaram que os maiores surtos da doença ocorreram devido à ocorrência de chuvas fortes e inundações. É importante ressaltar, no entanto, que Ávila-Pires ${ }^{29}$ defende a hipótese de que, nos períodos das enchentes, há uma maior sensibilidade para o diagnóstico da doença, o que poderia criar um artifício em relacionar o elevado número de casos confirmados da doença com altos índices pluviométricos.

Oliveira et al. ${ }^{30}$ realizaram um estudo onde foi sistematizado um modelo produtivo para a leptospirose, considerando os determinantes sociais, demográficos, biológicos e climáticos envolvidos na gênese da doença e os desafios para o seu con- 
trole. Entre os fatores ambientais listados pelos autores, podemos citar, entre outros: hidrografia, tipo de solo, temperatura, umidade, desastres naturais: inundações e enchentes. Entre os fatores sociodemográficos estão: densidade populacional, renda, escolaridade, ocupação, moradia, dentre outros. O presente estudo não considerou a análise de determinantes sociais da doença. Entretanto, mostra que a leptospirose possui uma dispersão desigual no espaço, evidenciada pelas diferentes taxas pelas Áreas de Planejamento do Município.

Todos estes estudos mostram uma associação entre a ocorrência de casos de leptospirose e de enchentes ou inundações. Este estudo indica que a média mensal de precipitações pode se constituir em um indicador que permita a realização de ações visando à preparação do setor saúde para o provável aumento de casos desta doença. Nas zonas urbanas, este fenômeno é potencializado pelo maior aglomerado populacional associado a uma desorganização da ocupação do espaço e uma oferta dos serviços de limpeza e saneamento inadequados. Os casos de leptospirose estudados no município do Rio de Janeiro mostram um padrão de temporalidade em relação à média mensal de precipitações. O número de casos começa a aumentar cerca de 1 mês após o período de chuvas, e se concentra principalmente em algumas áreas de planejamento. Esta característica permite o planejamento de ações pelos gestores, tanto de caráter preventivo quanto para melhorar o aporte de recursos diagnósticos e de tratamento para as comunidades expostas. Além disso, pode-se aventar a possibilidade de medidas que permitam a apropriação desta informação pela população, de forma a permitir que esta tome as precauções necessárias e procure atendimento de saúde imediato caso sinta necessidade.

Paralelamente, o trabalho de Oliveira et al. ${ }^{30}$ remete à possibilidade de construção de uma matriz de indicadores de saúde ambiental. Segundo Corvalan et al. ${ }^{31}$ entre as definições de indicadores estaria a possibilidade de eles se constituírem como uma informação útil, a partir da organização dos dados existentes, a ser utilizada como ferramenta pelos gestores.

Segundo Netto et al. ${ }^{32}$ o Ministério da Saúde do Brasil tem trabalhado com uma matriz de indicadores de saúde ambiental, tendo por base não só o impacto sobre a saúde causado por mudanças na situação ambiental mas, também, na compreensão do quadro socioeconômico. Assim, tem adotado o modelo denominado Força Motriz - Pressão - Situação - Exposição - Efeito Ações (FPSEEA). A “Força Motriz" refere-se às condições macroeconômicas que geram "Pressões" sobre o ambiente que determinam mudanças da "Situação" deste ambiente. Isto causa diferentes padrões de "Exposição" de populações humanas a esta situação do ambiente e um consequente "Efeito" sobre a saúde. Esta "cadeia" de condições deve determinar "Ações" a serem adotadas em cada nível identificado ${ }^{32}$.

Assim sendo, poder-se-ia propor uma matriz onde o modelo de desenvolvimento econômico, determinando uma urbanização acelerada e desordenada e o crescimento populacional, seriam as Forças motrizes. Estas, por sua vez, propiciariam a criação de aglomerados urbanos com crescente geração de resíduos que funcionariam como Pressão sobre a Situação do ambiente local. Estas condições de Pressão atuariam modificando a hidrografia, assoreando rios, destruindo a vegetação e alterando a capacidade de drenagem das águas, pela ausência ou inadequação das redes de água, esgoto e de coleta de lixo existentes. Esta Situação alterada do ambiente se refletiria em um Efeito sobre a saúde humana quando as pessoas são Expostas à água contaminada pelo agente biológico devido à ocorrência de enchentes ou inundações. A média mensal de precipitações poderia ser considerada como um dos indicadores de exposição que permitiria a adoção de ações visando à preparação do setor saúde para um aumento da ocorrência de casos, uma vez que contribui para o aumento de pessoas expostas à água contaminada ${ }^{33}$.

O presente estudo possui as limitações típicas de um estudo ecológico, como a qualidade e a disponibilidade dos dados. Entretanto, possui como grande vantagem o fato de se obter um tipo de modelagem onde é possível obter estimativas de risco a partir de variáveis ecológicas, o que é incomum em estudos ecológicos, que em geral se limitam a estimar correlações, sem estabelecer uma relação de causalidade.

Naturalmente, as ações a serem desencadeadas não devem estar direcionadas somente para o "Efeito", ou seja, o atendimento aos casos diagnosticados da doença. Ações realmente efetivas devem estar voltadas para os níveis mais altos desta matriz, com intervenções sobre as pressões que determinam as alterações do ambiente favorecendo o acúmulo de água contaminada. Assim, a construção de redes de água e esgoto adequadas, a desobstrução dos leitos dos rios e córregos, a adoção de um sistema de coleta e reaproveitamento de lixo para evitar a proliferação de roedores, enfim a organização de uma proposta de planejamento urbano seriam ações com real 
potencial de resolução sobre os determinantes da ocorrência de casos de lepstopirose no município do Rio de Janeiro. Para tal, considera-se imprescindível que as ações de mitigação considerem os indicadores de saneamento e de educação, como uma tentativa de captar as diferenças sociais colocadas, e que descrevem uma diferença na vulnerabilidade das populações expostas ao risco ${ }^{34}$. Além disso, é vital considerar, acerca da formulação de políticas públicas, diferentes olhares disciplinares para a efetividade das ações propostas ${ }^{35}$.

\section{Conclusão}

A transmissão da leptospirose envolve inúmeros fatores, incluindo o impacto do clima, cujo papel ainda não é totalmente compreendido. Elucidar o papel do clima é fundamental, pois facilita a análise do risco de epidemias e auxilia os esforços preventivos. Este estudo propõe um modelo para a análise da série histórica de leptospirose considerando a ocorrência de desastres naturais por inundação no Rio de Janeiro, fenômeno que ocorre com certa regularidade na cidade. A precipitação-lag mês mostrou ser um fator explicativo forte para o número de casos de leptospirose. Cabe, portanto, a articulação entre o trabalho da vigilância epidemiológica, especialmente aquele feito pelas salas de situação em períodos de crise, e as instâncias de gestão de riscos da vigilância ambiental, para aumentar a capacidade de resposta a desastres naturais no município do Rio de Janeiro.

\section{Colaboradores}

RM Guimarães, OG Cruz e VG Parreira participaram da concepção, análise de dados redação e revisão final do artigo; JD Vieira e ML Mazoto participaram da redação do artigo, CIRF Asmus participou da revisão final do artigo. 


\section{Referências}

1. Brasil. Ministério da Saúde (MS).Secretaria de Vigilância em Saúde. Departamento de Vigilância Epidemiológica. Doenças infecciosas e parasitárias : guia de bolso.

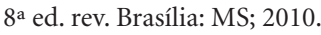

2. Tassinari WS, Pellegrini DC, Sá CB, Reis RB, Ko AI, Carvalho MS. Space-time clusters of urban leptospirosis. Trop Med Int Health 2008; 13(4):503-512.

3. Aleixo NCR, Neto JLS. Eventos pluviométricos extremos e saúde: perspectivas de interação pelos casos de leptospirose em ambiente urbano. Rev Brasileira de Geografia Médica e da Saúde 2010; 6(11):118-132.

4. Brasil. Ministério da Saúde (MS). Secretaria de Vigilância em Saúde. Departamento de Vigilância Epidemiológica. Guia de vigilância epidemiológica. $7^{\mathrm{a}}$ ed. Brasília: MS; 2009.

5. Genovez ME. Leptospirose: Uma doença de ocorrência além da época das chuvas. Biológico 2009; 71(1):1-3.

6. Nel JL, Le Maitre DC, Nel DC, Reyers B, Archibald S, van Wilgen BW, Forsyth GG, Theron AK, O'Farrell PJ, Kahinda JM, Engelbrecht FA, Kapangaziwiri E, van Niekerk L, Barwell L. Natural hazards in a changing world: a case for ecosystem-based management. PLoS One 2014; 9(5):e95942.

7. Freitas CM, Ximenes EF. Enchentes e saúde pública: uma questão na literatura científica recente das causas, consequências e respostas para prevenção e mitigação. Cien Saude Colet 2012; 17(6):1601-1616.

8. Tassinari WS, Pellegrini DCP, Sabroza PC, Carvalho MS. Distribuição espacial da leptospirose no Município do Rio de Janeiro, Brasil, ao longo dos anos de 1996-1999. Cad Saude Publica 2004; 20(6):364-372.

9. Brasil. Ministério do Meio Ambiente (MMA).Vulnerabilidade Ambiental - desastres naturais ou fenômenos induzidos? Brasília: MMA; 2007.

10. Soares TSP, Latorre MRDO, Laporta GZ, Buzzar MR. Análise espacial e sazonal da leptospirose no município de São Paulo, SP, 1998 a 2006. Rev Saude Publica 2010; 44(2):687-694.

11. Instituto Brasileiro de Geografia e Estatística (IBGE). Infográficos: Dados gerais do município do Rio de Janeiro. [acessado 2013 nov 18].Disponível em: http://www. ibge.com.br/cidadesat/painel/painel.php?lang $=\& \operatorname{cod}$ mun=330455\&search=rio-de-janeiro|rio-de-janeiro| infograficos:-dados-gerais-do-municipio

12. Prefeitura do Rio de Janeiro. Secretaria Municipal de Saúde (SMS). Leptospirose - Série Histórica. [acessado 2013 nov 20]. Disponível em: http://www.rio.rj.gov.br/ web/sms

13. Brasil. Ministério da Saúde (MS). Departamento de Informática do SUS. [acessado 2013 nov 20]. Disponível em: http://www.datasus.gov.br

14. Prefeitura do Rio de Janeiro. Secretaria Municipal de Obras (SMO). Precipitação mensal Acumulada. [acessado 2013 nov 20]. Disponível em: http://www.rio.rj.gov. $\mathrm{br} / \mathrm{web} / \mathrm{smo}$

15. Instituto Pereira Passos. Bases Cartográficas Digitais do Município do Rio de Janeiro. [acessado 2013 nov 20]. Disponível em: http://ipprio.rio.rj.gov.br/rio-em-dados /mapoteca

16. McCullagh P, Nelder JA. Generalized linear models. London: Chapman and Hall; 1989.

17. Venables WN, Ripley BD. Modern Applied Statistics with S. $4^{\text {th }}$ ed. New York: Springer; 2002.
18. Bivand R, Lewin-Koh N. Maptools: Tools for reading and handling spatial objects. R package version 0.8-27. [acessado 2013 nov 22]. Disponível em: http://CRAN. R-project.org $/$ package $=$ maptools

19. Magalhães GB, Zanella ME, Sales MCL. A ocorrência de chuvas e a incidência de leptospirose em FortalezaCE. Rev Brasileira de Geografia Médica e Saúde 2009; 5(9):77-87.

20. Figueiredo CM, Morais AC, Oliveira MAA, Alves WR, Ooteman MC, Chamone CB, Koury MC. Leptospirose humana no município de Belo Horizonte Minas Gerais, Brasil: uma abordagem geográfica. Rev Soc Bras Med Trop 2001; 34(4):331-338.

21. Barcellos C, Sabroza PC. The place behind the case: leptospirosis risks and associated environmental conditions in a flood-related outbreak in Rio de Janeiro. Cad Saude Publica 2001; 17(Supl.1):59-67.

22. Sobral A, Freitas CM, Andrade EV, Lyra GFD, Mascarenhas MS, Alencar MRF, Castro RAL, França RF. Desastres naturais - sistemas de informação e vigilância: uma revisão da literatura. Epidemiol. Serv. Saúde 2010; 19(4):389-402.

23. Pellegrini DCP. Análise espaço-temporal da leptospirose no município do Rio de Janeiro [dissertação]. Rio de Janeiro: Fiocruz; 2002.

24. Barcellos C, Lammerhirt CB, Almeida MAB, Santos E. Distribuição espacial da Leptospirose no Rio Grande do Sul, Brasil. Recuperando a ecologia dos estados ecológicos. Cad Saude Publ 2003;19(5):1283-1292.

25. Paula VE. Leptospirose humana: uma análise climato-geográfica de sua manifestação no Brasil, Paraná e Curitiba. In: Anais XII Simpósio Brasileiro de Sensoriamento Remoto; 16-21 abril 2005; Goiânia, Brasil. INPE. p. 2301-2308.

26. Souza MHF, Aarão Junior RNN, Costa ACL. Leptospirose humana: uma análise de sua manifestação no município de Belém/PA. In: XVI Congresso Brasileiro de Meteorologia. Belém/PA, 2010. [acessado 2013 nov 23]. Disponível em: http://www.cbmet2010.com/anais/ artigos/308_94172.pdf

27. Scolovschi C, Angelakis E, Renvoisé A. Strikes, flooding, rats, and leptospirosis in Marseille, France. Int $J$ Infect Dis 2011; 15(10):e710-e715.

28. Zhang C, Wang H, Yan J. Leptospirosis prevalence in Chinese populations in the last two decades. Microbes Infect 2012; 14(4):317-323.

29. Avila-Pires FD. Leptospirose e enchentes: uma falsa correlação? Revista de Patologia Tropical 2006; 35(3): 199-204.

30. Oliveira DSC, Guimarães MJB, Medeiros Z. Modelo produtivo para a Leptospirose. Revista de Patologia Tropical 2009; 38(1):17-26.

31. Corvalán C, Briggs D, Zielhuis G. The need for information: environmental health indicators. In: Corvalán C, Briggs D, Zielhuis G, editors. Decision-making in environmental health: from evidence to action. London: $\mathrm{E}$ \& FN Spon; 2000. p. 25-51.

32. Netto GF, Freitas CM, Andahur JP, Pedroso MM, Rohlfs DB. Impactos socioambientais na situação de saúde da população brasileira: Estudo de indicadores relacionados ao saneamento ambiental inadequado. Tempus. Actas em Saúde Coletiva 2009; 4(4):53-71. 
33. Corvalán C, Kjellström T. A Health-and-Environment Cause-Effect Framework. In: Pastides H, Corvalán C. Methods for Health Impact in Environmental and $\mathrm{Oc}$ cupational Health. Geneva: WHO;1997. (Report of a WHO/ILO Consultation. July-97).

34. Carneiro FF, Franco Netto G, Corvalan C, Freitas CM, Sales LBF. Saúde ambiental e desigualdades: construindo indicadores para o desenvolvimento sustentável. Cien Saude Colet 2012; 17(6):1419-1425.

35. Weihs M, Mertens F. Os desafios da geração do conhecimento em saúde ambiental: uma perspectiva ecossistêmica. Cien Saude Colet 2013; 18(8):1501-1510.

Artigo apresentado em 29/03/2014

Aprovado em 07/06/2014

Versão final apresentada em 09/06/2014 